Pacific Journal of Mathematics

CRITERIA FOR OSCILLATORY SUBLINEAR SCHRÖDINGER 


\title{
CRITERIA FOR OSCILLATORY SUBLINEAR SCHRÖDINGER EQUATIONS
}

\author{
C. A. Swanson
}

The semilinear Schrödinger equation

$$
L u \equiv \Delta u+f(x, u)=0, \quad x \in \Omega
$$

will be considered in an exterior domain $\Omega \subset R^{n}, n \geq 2$, where $f$ is nonnegative and locally Hölder continuous in $\Omega \times(0, \infty)$. One objective is to find sharp necessary conditions for (1) to be oscillatory in $\Omega$ under the sublinear hypothesis that $\max _{|x|=r} t^{-1} f(x, t)$ is a nonincreasing function of $t$ in $(0, \infty)$ for each fixed $r>0$. The necessary conditions below are proved in $\S 2$ :

$$
\begin{array}{ll}
\int^{\infty} r \max _{|x|=r} f(x, c \log r) d r=+\infty & \text { if } n=2 ; \\
\int^{\infty} r \max _{|x|=r} f(x, c) d r=+\infty & \text { if } n \geq 3
\end{array}
$$

for some positive constant $c$. Sufficient conditions for (1) to be oscillatory in $\Omega$ are proved in $\$ 3$ under a modified sublinear hypothesis. These results are then combined to yield characterizations of oscillatory sublinear equations of the Emden-Fowler type in exterior domains.

The sublinear Emden-Fowler (or Lane-Emden) equation is the prototype

$$
\Delta u+p(x)|u|^{\gamma} \operatorname{sgn} u=0, \quad 0<\gamma<1, x \in \Omega,
$$

where $p(x)$ is nonnegative and locally Hölder continuous in $\Omega$. A theorem of Kitamura and Kusano [7] states in particular that (2) is oscillatory in a exterior domain $\Omega$ in $R^{n}, n \geq 2$, if

$$
\int^{\infty} r P_{1}(r) d r=+\infty
$$

where $P_{1}(r)=\min _{|x|=r} p(x)$. The same is true if $P_{1}(r)$ is replaced by the spherical mean of $p(x)$ over the sphere of radius $r$ (see §3). Under additional regularity hypotheses on $p(x)$ it was proved by $\mathrm{E}$. S. Noussair and the writer [12] that (3) is in fact necessary and sufficient for (2) to be oscillatory in $\Omega \subset R^{n}$ if $n \geq 3$. However, this is not so if $n=2$; an easy counterexample is provided in the case that (2) is a radial equation:

$$
\frac{1}{r} \frac{d}{d r}\left(r \frac{d u}{d r}\right)+p(r)|u|^{\gamma} \operatorname{sgn} u=0
$$


where $p(r)=r^{-2}(\log r)^{-\delta}, 1<\delta<1+\gamma, r>0$. Liouville's transformation $r=e^{s}, h(s)=u\left(e^{s}\right)$ sends (4) into the canonical form

$$
h^{\prime \prime}(s)+e^{2 s} p\left(e^{s}\right)|h(s)|^{\gamma} \operatorname{sgn} h(s)=0,
$$

which is oscillatory at $s=\infty$ if and only if

$$
\int^{\infty} s^{\gamma} e^{2 s} p\left(e^{s}\right) d s=+\infty
$$

by Belohorec's well-known theorem [2], or equivalently, if and only if

$$
\int^{\infty} r(\log r)^{\gamma} p(r) d r=+\infty
$$

In the present example, (5) is satisfied while (3) fails. Therefore (3) is not necessary for oscillation of (2) in 2 dimensions. It might be expected that a necessary condition for oscillation of (2) (or (1)) is similar to (5), and in fact Theorem 2.4 below shows, under the hypotheses that $p(x)$ is nonnegative and Hölder continuous, that a necessary condition for (2) to be oscillatory in an exterior domain $\Omega \subset R^{2}$ is

$$
\int^{\infty} r(\log r)^{\gamma}\left[\max _{|x|=r} p(x)\right] d r=+\infty
$$

One of our main objectives is to improve (3) in $R^{2}$; this is accomplished in Theorems 3.3 and 3.4. In $\$ 2$ the necessary conditions for oscillation mentioned above (i.e. the nonoscillation results) are extended to the general sublinear case (1). Theorem 3.6 and Corollary 3.7 contain characterizations of oscillatory sublinear equations (1) or (2) in $R^{n}, n \geq 3$, under suitable regularity hypotheses on $f(x, u)$ or $p(x)$.

2. Necessary conditions for oscillation. Points in Euclidean $n$-space $R^{n}$ are denoted by $x=\left(x_{1}, \ldots, x_{n}\right)$, and the Euclidean length of $x$ is written $|x|$. The following notation will be used throughout the sequel:

$$
\begin{aligned}
& S_{a}=\left\{x \in R^{n}:|x|=a\right\}, \quad a>0 ; \\
& G_{a}=\left\{x \in R^{n}:|x|>a\right\} .
\end{aligned}
$$

An exterior domain $\Omega$ in $R^{n}$ is defined by the property that $G_{a} \subset \Omega$ for some $a>0$. 
For a bounded domain $M \subset R^{n}$, the Hölder norms of a function $u: \bar{M} \rightarrow R^{1}$ are defined by

$$
\begin{aligned}
\|u\|_{\lambda, \bar{M}}= & \sup _{\substack{x, y \in \bar{M} \\
x \neq y}} \frac{|u(x)-u(y)|}{|x-y|^{\lambda}} \\
\|u\|_{m+\lambda, \bar{M}}= & \sum_{|i|=m}\left\|D^{i} u\right\|_{\lambda, \bar{M}}+\sum_{|i| \leq m} \sup _{x \in \bar{M}}\left|D^{i} u(x)\right|, \\
& 0<\lambda<1, \quad m=1,2, \ldots,
\end{aligned}
$$

where $i$ denotes a multi-index of length $|i|$. The Hölder space $C^{m+\lambda}(\bar{M})$ is defined as the set of all continuous real-valued functions on $\bar{M}$ with partial derivatives on $\bar{M}$ such that $\|u\|_{m+\lambda, \bar{M}}$ is finite, $0<\lambda<1, m=$ $0,1, \ldots$ The notation $C_{\mathrm{loc}}^{m+\lambda}(\Omega)$ denotes the set of all $u: \Omega \rightarrow R$ such that $u \in C^{m+\lambda}(\bar{M})$ for every bounded domain $M \subset \Omega$. The notation $C_{\mathrm{loc}}^{m+\lambda}\left(\Omega \times R^{+}\right)$is defined similarly, where $R^{+}=(0, \infty)$.

Equation (1) is to be considered in an exterior domain $\Omega \subset R^{n}$ subject to the assumptions below.

\section{ASSUMPTIONS}

(A) $f \in C_{\text {loc }}^{\lambda}\left(\Omega \times R^{+}\right)$for some $\lambda$ in $0<\lambda<1$, fixed in the sequel.

(B) $0 \leq f(x, t) \leq \operatorname{tg}(|x|, t)$ for all $x \in \Omega$ and for all $t>0$, where $g \in C_{\mathrm{loc}}^{\lambda}\left(R^{+} \times R^{+}\right)$and $g(r, t)$ is a nonincreasing function of $t$ in $R^{+}$for each fixed $r>0$.

The above nonincreasing property of $g(r, t)$ is a sublinear condition for equation (1). For example, assumptions (A) and (B) hold in the Emden-Fowler prototype (2), i.e. $f(x, t)=p(x) t^{\gamma}, 0<\gamma<1$, where $p(x)$ is nonnegative in $\Omega$ and $p \in C_{\mathrm{loc}}^{\lambda}(\Omega)$. In this case an example of a function $g$ in $(B)$ is

$$
g(r, t)=\left[\max _{|x|=r} p(x)\right] t^{\gamma-1} .
$$

A solution of $L u=0[L u \leq 0, L u \geq 0]$ in $\Omega$ is a function $u \in C_{\mathrm{loc}}^{2+\lambda}(\Omega)$, with $\lambda$ as in (A), such that $(L u)(x)=0[(L u)(x) \leq 0,(L u)(x) \geq 0$, respectively] for all $x \in \Omega$. The operator $L$ given by (1) is called oscillatory in $\Omega$ whenever every solution of (1) defined in $G_{a} \subset \Omega$ for some $a>0$ changes sign in $G_{r}$ for all $r \geq a$. Then $L$ is nonoscillatory in $\Omega$ whenever (1) has a positive solution $u(x)$ in $G_{b}$ for some $b \geq a$.

2.1. TheOREM. Let $L$ be the operator defined by (1) where $f$ is nonnegative and satisfies assumption (A) in an exterior domain $\Omega$, and suppose that $G_{a} \subset \Omega$ for some $a>0$. If there exists a positive solution $v$ and a nonnegative solution $w$ of $L v \leq 0$ and $L w \geq 0$, respectively, in $G_{a}$ such that $w(x) \leq$ $v(x)$ throughout $G_{a} \cup S_{a}$, then equation (1) has at least one solution $u(x)$ satisfying $u(x)=v(x)$ on $S_{a}$ and $w(x) \leq u(x) \leq v(x)$ throughout $G_{a}$. 
A proof was given by Noussair and the writer [12]. A variation with an additional monotony hypothesis appears in [11]. Versions of this theorem for bounded domains appear in the works of Nagumo [9], Cohen [5], Keller [6], Simpson and Cohen [13], and Amann [1].

The corollary below applies to the case that the ordinary differential equation

$$
\frac{d}{d r}\left(r^{n-1} \frac{d \zeta}{d r}\right)+r^{n-1} \zeta g(r, \zeta)=0
$$

has a positive solution $\zeta \in C^{2+\lambda}[a, b]$ for some $a>0$ and for all $b>a$.

2.2. Corollary. If (A) and (B) hold, equation (1) is nonoscillatory in an exterior domain $\Omega \subset R^{n}, n \geq 2$, if (7) has a positive solution $\zeta(r)$ in $a<r<\infty$ for some $a>0$ such that $\zeta \in C^{2+\lambda}[a, b]$ for all $b>a$.

Proof. Let $v$ be the function defined in $G_{a}$ by $v(x)=\zeta(r), r=|x| \geq a$. Then

$$
\begin{aligned}
r^{n-1}(L v)(x) & =\frac{d}{d r}\left(r^{n-1} \frac{d \zeta}{d r}\right)+r^{n-1} f(x, v(x)) \\
& \leq \frac{d}{d r}\left(r^{n-1} \frac{d \zeta}{d r}\right)+r^{n-1} \zeta(r) g(r, \zeta(r)),
\end{aligned}
$$

and hence $(L v)(x) \leq 0$ for all $x \in G_{a}$ by (7). Since $w(x) \equiv 0$ satisfies $(L w)(x) \geq 0$, Theorem 2.1 shows that (1) has a solution $u(x)$ satisfying $0 \leq u(x) \leq v(x)=\zeta(r)$ for $|x| \geq a$. However, $(\Delta u)(x) \leq 0$ in the annulus $G_{a, b}=\left\{x \in R^{n}: a<|x|<b\right\}, u(x)=v(x)>0$ for $|x|=a$, and $u(x) \geq 0$ for $|x|=b$, and therefore $u(x)>0$ throughout $G_{a, b}$ by the maximum principle. Since $b$ is arbitrary, $u(x)$ is a positive solution of (1) in $G_{a}$.

To apply Corollary 2.2, we shall appeal to the following theorem of Belohorec [3, Theorem 3], Coffman and Wong [4, Theorem 2], concerning the ordinary differential equation

$$
\frac{d^{2} u}{d t^{2}}+u g(t, u)=0, \quad 0<t<\infty
$$

2.3. TheOREM (Belohorec, Coffman and Wong). Let $f(t, u)=u g(t, u)$ be continuous and nonnegative for $0<t<\infty, 0<u<\infty$, and suppose that $g(t, u)$ is nonincreasing in $u$ for each $t$. Then equation (8) has an unbounded positive solution $u(t)$ in $(a, \infty)$ for some $a>0$ if and only if

$$
\int^{\infty} \operatorname{tg}(t, c t) d t<\infty
$$

for some $c>0$. 
Although not relevant here, it is proved in [3] and [4] that (9) characterizes equations (8) possessing an asymptotically linear unbounded solution.

2.4. THEOREM. Under assumptions (A) and (B), (1) has a positive solution $u(x)$ in an exterior domain $G_{a} \subset R^{n}$ for some $a>0$ if

$$
\begin{gathered}
\int^{\infty} r \log r g(r, c \log r) d r<\infty \quad(n=2) \\
\int^{\infty} r g(r, c) d r<\infty \quad(n \geq 3)
\end{gathered}
$$

for some $c>0$.

Proof. If $n=2$, Liouville's transformation $r=e^{s}, h(s)=\zeta\left(e^{s}\right)$ changes (7) into the standard form

$$
h^{\prime \prime}(s)+e^{2 s} h(s) g\left(e^{s}, h(s)\right)=0 .
$$

By Theorem 2.3, (12) has a positive solution $h(s)$ in some interval $(A, \infty)$ if and only if

$$
\int^{\infty} s e^{2 s} g\left(e^{s}, c s\right) d s<\infty
$$

for some $c>0$, which is equivalent to (10). Since $g \in C^{\lambda}$ by assumption (B), standard regularity theorems (see e.g. [8]) show that $h \in C^{2+\lambda}[A, B]$ for all $B>A$, or equivalently $\zeta \in C^{2+\lambda}[a, b]$ for all $b>a=e^{A}$. Then Corollary 2.2 shows that (10) is sufficient for (1) to have a positive solution $u(x)$ in $G_{a} \subset R^{2}$ for some $a>0$.

Similarly if $n \geq 3$ the change of variables

$$
r=\beta(s)=(\nu s)^{\nu}, \quad h(s)=s \zeta(\beta(s)),
$$

where $\nu=1 /(n-2)$, transforms (7) into

$$
h^{\prime \prime}(s)+s^{-4}[\beta(s)]^{2 n-2} h(s) g\left(\beta(s), \frac{h(s)}{s}\right)=0 .
$$

By Theorem 2.3, (13) has a positive solution in some interval $(A, \infty)$ if and only if

$$
\int^{\infty} s^{-3}[\beta(s)]^{2 n-2} g(\beta(s), c) d s<\infty
$$

for some $c>0$, establishing the sufficiency of (11) for (1) to have a positive solution in $G_{a} \subset R^{n}, n \geq 3$, for some $a>0$. 
In the special case (2) of (1), i.e. $f(x, u)=p(x) u^{\gamma}, 0<\gamma<1$, where $p(x)$ is nonnegative in $\Omega$ and $p \in C_{\mathrm{loc}}^{\lambda}(\Omega)$, we choose $g(r, u)$ in (B) to be $P_{2}(r) u^{\gamma-1}$, where

$$
P_{2}(r)=\max _{|x|=r} p(x) .
$$

In this case, conditions (10) and (11) reduce to, respectively

$$
\begin{gathered}
\int^{\infty} r(\log r)^{\gamma} P_{2}(r) d r<\infty, \quad n=2 \\
\int^{\infty} r P_{2}(r) d r<\infty, \quad n \geq 3 .
\end{gathered}
$$

3. Sufficient conditions for oscillation. Oscillation criteria for (1) or (2) will be generated by developing necessary conditions for (1) or (2) to have a positive solution $u(x)$ in some exterior domain $G_{a}, a>0$, under the following alternative to assumption (B):

\section{ASSUMPTION}

(C) $f(x, u) \geq p(x) \phi(u)$ for all $x \in \Omega$ and for all $u \geq 0$, where $p$ is continuous and nonnegative in $\Omega ; \phi \in C^{1}[0, \infty) ; \phi(u)>0, \phi^{\prime}(u)>0$, and $\Phi(u)<\infty$ for all $u>0$, where

$$
\Phi(u)=\int_{0}^{u} \frac{d t}{\phi(t)}, \quad u>0 .
$$

The sublinear condition $\Phi(u)<\infty$ is satisfied, for example, if $\phi(u)$ $=|u|^{\gamma} \operatorname{sgn} u, 0<\gamma<1$. A solution of (1) is now a classical solution, i.e. $u \in C^{2}(\Omega)$ and $L u=0$ at every point in $\Omega$.

The extension of (1) to negative $u$ can be made by adjoining the conditions $f(x,-u)=-f(x, u)$ and $\phi(-u)=-\phi(u)$ for all $u>0$, or weaker requirements. Then the existence of a negative solution $u(x)$ of (1) is equivalent to the existence of a positive solution, and our theorems imply criteria for the nonexistence of any one-signed solutions.

3.1. LEMMA. If $u$ is a positive solution of (1) in $G_{a}$ for some $a>0$, and $z \in C^{2}\left(G_{a}\right)$ is an arbitrarypositive function in $G_{a}$, then $\Phi(u)$ satisfies the differential inequality

$$
\begin{aligned}
& -\Delta[z(x) \Phi(u(x))] \\
& \quad \geq z(x) p(x)-\Phi(u(x))(\Delta z)(x)-\frac{|(\nabla z)(x)|^{2}}{z(x) \phi^{\prime}(u(x))}
\end{aligned}
$$

for all $x \in G_{a}$. 
Proof. An easy calculation on the basis of (1), (16), and assumption (C) shows that

$$
\Delta \Phi(u) \leq-p-\phi^{\prime}(u)|\nabla \Phi(u)|^{2}
$$

in $G_{a}$, from which

$$
\begin{gathered}
-\Delta(z \Phi(u)) \geq z p+z \phi^{\prime}(u)\left|\nabla \Phi(u)-\left[z \phi^{\prime}(u)\right]^{-1} \nabla z\right|^{2} \\
-\Phi(u) \Delta z-\left[z \phi^{\prime}(u)\right]^{-1}|\nabla z|^{2},
\end{gathered}
$$

implying (17) since $\phi^{\prime}(u)>0$ for $u>0$.

The next lemma is a specialization to the case $n=2, z(x)=\phi(h)$, $h=\log |x|$.

3.2. LEMMA. If $u$ is a positive solution of (1) in $G_{a} \subset R^{2}$ for some a $>0$, then

$$
-\Delta[\phi(h) \Phi(u)] \geq \phi(h) p(x)-\frac{\left[\phi^{\prime}(h)\right]^{2}+\phi(h) \phi^{\prime \prime}(h) \Phi(u) \phi^{\prime}(u)}{r^{2} \phi(h) \phi^{\prime}(u)}
$$

where $h=\log r, r=|x| \geq a$.

Proof. If $z(x)=\phi(h)$, then

$$
\nabla z=\frac{\phi^{\prime}(h) x}{r^{2}}, \quad \Delta z=\frac{\phi^{\prime \prime}(h)}{r^{2}}
$$

and (17) reduces to (18).

In the classical sublinear case, $\phi(u)=u^{\gamma}, 0<\gamma<1, \Phi(u) \phi^{\prime}(u)=$ $\gamma /(1-\gamma)$, and hence

$$
\left[\phi^{\prime}(h)\right]^{2}+\phi(h) \phi^{\prime \prime}(h) \Phi(u) \phi^{\prime}(u) \equiv 0 .
$$

Then (18) simplifies to

$$
-\Delta w(x) \geq(\log r)^{\gamma} p(x), \quad r=|x| \geq a
$$

where

$$
w(x)=(1-\gamma)^{-1}(\log r)^{\gamma}[u(x)]^{1-\gamma} .
$$

The spherical mean $U(r)=m(r ; u)$ of a function $u: R^{n} \rightarrow R^{l}$ over the sphere $S_{r}$ of radius $r$ is defined by

$$
m(r ; u)=\frac{1}{s\left(S_{r}\right)} \int_{S_{r}} u(x) d s=\frac{1}{\omega\left(S_{1}\right)} \int_{S_{1}} u(x) d \omega,
$$

where $s$ and $\omega$ denote the measure on $S_{r}$ and $S_{1}$, respectively; $d s=r^{n-1} d \omega$. 
3.3. ThEOREM. If assumption (C) holds where $\phi(u)=u^{\gamma}, 0<\gamma<1, a$ necessary condition for (1) to have a positive solution $u(x)$ in some exterior domain $G_{a} \subset R^{2}$ is

$$
\int^{\infty} r(\log r)^{\gamma} m(r ; p) d r<\infty
$$

Proof. The spherical mean of any function $w \in C^{2}\left(G_{a}\right)$ satisfies [10; Lemma 2, pp. 69-70]

$$
\frac{d}{d r}\left[r^{n-1} \frac{d m(r ; w)}{d r}\right]=\frac{r^{n-1}}{\omega\left(S_{1}\right)} \int_{S_{1}} \Delta w(x) d \omega .
$$

For $n=2,(19),(20)$ and (22) imply the differential inequality

$$
-\frac{d}{d r}\left[r \frac{d m(r ; w)}{d r}\right] \geq r(\log r)^{\gamma} m(r ; p)
$$

for $a<r<\infty$. Define $W(r)=m(r ; w)$. Then $Z(r)=r W^{\prime}(r)$ is nonincreasing for $r>a$ by (23). Since $w(x)>0$ by hypothesis, so also $W(r)>0$ by (20), it follows that $W^{\prime}(r)>0$ for all $r>a$; for if

$$
W(r)-W(R)=\int_{R}^{r} \frac{Z(t)}{t} d t \leq Z(R) \log \frac{r}{R},
$$

contradicting the positivity of $W(r)$ for all $r>a$. Integration of (23) over $(a, r)$ gives

$$
-r W^{\prime}(r)+a W^{\prime}(a) \geq \int_{a}^{r} t(\log t)^{\gamma} m(t ; p) d t .
$$

Since $W^{\prime}(r)>0$ for all $r>a$, (24) implies the conclusion (21) of Theorem 3.3.

Comparison of (21) with (10) or (14) indicates the sharpness of these criteria. In fact, as the theorem below states, condition (21) characterizes equations (2) possessing a positive solution in some exterior domain in $R^{2}$ provided the condition

$$
\limsup _{r \rightarrow \infty} \frac{P_{2}(r)}{m(r ; p)}<\infty, \quad P_{2}(r)=\max _{|x|=r} p(x)
$$

is added to the other hypotheses.

3.4. THEOREM. Suppose that $p(x)$ in (2) is nonnegative in an exterior domain $\Omega$ in $R^{2}, p \in C_{\mathrm{loc}}^{\lambda}(\Omega)$, and (25) is satisfied. Then (21) is necessary and sufficient for equation (2) to have a positive solution in some exterior domain in $R^{2}$. 
Proof. The necessity of (21) is the content of Theorem 3.3 and the sufficiency follows from (14) and (25).

The theorem below is a slight modification of a theorem of Kitamura and Kusano [7], with an alternative proof which seems more direct and elementary.

3.5. THEOREM (Kitamura and Kusano). If assumption (C) holds, a necessary condition for (1) to have a positive solution in some exterior domain in $R^{n}, n \geq 2$, is

$$
\int^{\infty} r m(r ; p) d r<\infty
$$

Proof. If $u(x)$ is a positive solution of (1) in $G_{a}$ for some $a>0$, and $z(x) \equiv 1$ in Lemma 3.1, then (17) reduces to $-\Delta \Phi(u(x)) \geq p(x)$, and (22) implies the differential inequality

$$
-\frac{d}{d r}\left[r^{n-1} \frac{d W}{d r}\right] \geq r^{n-1} m(r ; p),
$$

where $W(r)=m(r ; \Phi(u))$. For $n \geq 3$ the change of variables

$$
r=\beta(s)=(\nu s)^{\nu}, \quad h(s)=s W(\beta(s)), \quad \nu=\frac{1}{n-2}
$$

transforms (27) into

$$
-h^{\prime \prime}(s) \geq s^{-3}[\beta(s)]^{2 n-2} m(\beta(s) ; p) .
$$

Integration over $\left(A, s_{0}\right)$ gives

$$
-h^{\prime}\left(s_{0}\right)+h^{\prime}(A) \geq \nu \int_{a}^{r_{0}} r m(r ; p) d r,
$$

where $a=\beta(A), r_{0}=\beta\left(s_{0}\right)$. Since $h^{\prime}(s)$ is nonincreasing by (28) and $h(s)>0$ for $s>A, h^{\prime}\left(s_{0}\right)>0$ for all $s_{0}>A$ by a standard argument, and (26) follows from (29).

If $n=2$, (26) follows directly from integration of (27), but this is unnecessary because of the stronger conclusion (21) of Theorem 3.3.

Condition (26) characterizes equations (1) with a positive solution in some exterior domain in $R^{n}, n \geq 3$, provided the extra hypothesis

$$
\limsup _{r \rightarrow \infty} \frac{g(r, c)}{m(r ; p)}<\infty,
$$

for some constant $c>0$, is adjoined to hypotheses (B) and (C).

3.6 THEOREM. If (A), (B), (C), and (30) hold, then (26) is necessary and sufficient for (1) to have a positive solution in an exterior domain in $R^{n}$, $n \geq 3$. 
Proof. Theorem 3.5 establishes the necessity of (26), and Theorem 2.4 establishes the sufficiency in view of (30).

If (1) is specialized to (2), condition (30) reduces to (25) and the following corollary results.

3.7 CoROllaRy. Suppose that $p(x)$ is nonnegative in an exterior domain $\Omega$ in $R^{n}, n \geq 3, p \in C_{\mathrm{loc}}^{\lambda}(\Omega), 0<\lambda<1$, and (25) is satisfied. Then (26) is necessary and sufficient for (2) to have a positive solution in some exterior domain in $R^{n}, n \geq 3$.

An equivalent statement is that the condition

$$
\int^{\infty} r m(r ; p) d r=+\infty
$$

is necessary and sufficient for (2) to be oscillatory in $\Omega$, i.e. for every solution of (2) in $G_{a} \subset \Omega$ to change sign in $G_{r}$ for all $r \geq a$. The same applies to (1) under the hypotheses of Theorem 3.6 if the function $f$ in (1) is odd in $u$, i.e. $f(x,-u)=-f(x, u)$ for all $u>0$.

The original version of Theorem 3.3 contained a slightly weaker version of condition (21). I am grateful to Professor Takeshi Kura for supplying me with his typescript "Oscillation criteria for a class of semilinear elliptic equations of the second order", indicating the present sharp version.

Added in proof: A variant of Theorem 3.3 has recently been given by Hiroshi Onose, "Oscillation criteria for the sublinear Schrödinger equation”, Proc. Amer. Math. Soc., 85 (1982), 69-72.

\section{REFERENCES}

1. H. Amann, On the existence of positive solutions of nonlinear elliptic boundary value problems, Indiana Univ. Math. J., 21 (1971), 125-146.

2. S. Belohorec, Oscillatory solutions of certain nonlinear differential equations of second order, Mat.-Fyz. Casopis Sloven. Akad. Vied., 11 (1961), 250-255 (in Czech.).

3. Monotone and oscillatory solutions of a class of nonlinear differential equations, Ibid., 19 (1969), 169-187.

4. C. V. Coffman and J. S. W. Wong, Oscillation and nonoscillation theores for second order ordinary differential equations, Funkcial. Ekvac., 15 (1972), 119-130.

5. D. S. Cohen, Positive solutions of a class of nonlinear eigenvalue problems, J. Math. Mech., 17 (1967), 209-216.

6. H. B. Keller, Positive solutions of some non-linear eigenvalue problems, J. Math. Mech., 19 (1969), 279-295.

7. Y. Kitamura and T. Kusano, An oscillation theorem for a sublinear Schrödinger equation, Utilitas Math., 14 (1978), 171-175.

8. O. A. Ladyzhenskaya and N. N. Ural'tseva, Linear and Quasilinear Elliptic Equations, Academic Press, New York, 1968. 
9. M. Nagumo, On principally linear elliptic differential equations of the second order, Osaka Math. J., 6 (1954), 207-229.

10. E. S. Noussair and C. A. Swanson, Oscillation theory for semilinear Schrödinger equations and inequalities, Proc. Roy. Soc. Edinburgh, Sect. A, 75 (1975/76), 67-81.

11. Positive solutions of semilinear Schrödinger equations in exterior domains, Indiana Univ. Math. J., 28 (1979), 993-1003.

12. __ Positive solutions of quasilinear elliptic equations in exterior domains, J. Math. Anal. Appl., 75 (1980), 121-133.

13. R. B. Simpson and D. S. Cohen, Positive solutions for non-linear elliptic eigenvalue problems, J. Math. Mech., 19 (1970), 895-910.

Received September 18, 1980. Support from the Natural Sciences and Engineering Research Council of Canada is acknowledged with gratitude.

UNIVERSITY OF BRITISH COLUMBIA

VANCOUVER, B.C. V6T 1Y4, CANADA 



\title{
PACIFIC JOURNAL OF MATHEMATICS EDITORS
}

\author{
Donald BABBITT (Managing Editor) \\ University of California \\ Los Angeles, CA 90024 \\ Hugo Rossi \\ University of Utah \\ Salt Lake City, UT 84112 \\ C. C. Moore and Arthur Ogus \\ University of California \\ Berkeley, CA 94720
}

\author{
J. Dugundi \\ Department of Mathematics \\ University of Southern California \\ Los Angeles, CA 90089-1113
}

R. FinN and H. SAMELSON

Stanford University

Stanford, CA 94305

\section{ASSOCIATE EDITORS}
R. ARENS
E. F. BECKENBACH
B. H. NeumanN
F. WOLF
K. YoSHIDA (1906-1982)

\section{SUPPORTING INSTITUTIONS}

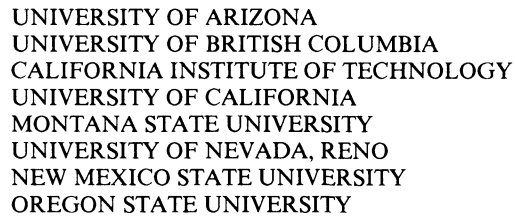

UNIVERSITY OF ARIZONA

UNIVERSITY OF BRITISH COLUMBIA

CALIFORNIA INSTITUTE OF TECHNOLOGY

UNIVERSITY OF CALIFORNIA

MONTANA STATE UNIVERSITY

UNIVERSITY OF NEVADA, RENO

NEW MEXICO STATE UNIVERSITY

OREGON STATE UNIVERSITY

\author{
UNIVERSITY OF OREGON \\ UNIVERSITY OF SOUTHERN CALIFORNIA \\ STANFORD UNIVERSITY \\ UNIVERSITY OF HAWAII \\ UNIVERSITY OF TOKYO \\ UNIVERSITY OF UTAH \\ WASHINGTON STATE UNIVERSITY \\ UNIVERSITY OF WASHINGTON
}

The Supporting Institutions listed above contribute to the cost of publication of this Journal, but they are not owners or publishers and have no responsibility for its content or policies.

Mathematical papers intended for publication in the Pacific Journal of Mathematics should be in typed form or offset-reproduced (not dittoed), double spaced with large margins. Please do not use built up fractions in the text of the manuscript. However, you may use them in the displayed equations. Underline Greek letters in red, German in green, and script in blue. The first paragraph must be capable of being used separately as a synopsis of the entire paper. In particular it should contain no bibliographic references. Please propose a heading for the odd numbered pages of less than 35 characters. Manuscripts, in triplicate, may be sent to any one of the editors. Please classify according to the scheme of Math. Reviews, Index to Vol. 39. Supply name and address of author to whom proofs should be sent. All other communications should be addressed to the managing editor, or Elaine Barth, University of California, Los Angeles, California 90024.

There are page-charges associated with articles appearing in the Pacific Journal of Mathematics. These charges are expected to be paid by the author's University, Government Agency or Company. If the author or authors do not have access to such Institutional support these charges are waived. Single authors will receive 50 free reprints; joint authors will receive a total of 100 free reprints. Additional copies may be obtained at cost in multiples of 50 .

The Pacific Journal of Mathematics is issued monthly as of January 1966. Regular subscription rate: $\$ 132.00$ a year (6 Vol., 12 issues). Special rate: $\$ 66.00$ a year to individual members of supporting institutions.

Subscriptions, orders for numbers issued in the last three calendar years, and changes of address should be sent to Pacific Journal of Mathematics, P.O. Box 969, Carmel Valley, CA 93924, U.S.A. Old back numbers obtainable from Kraus Periodicals Co., Route 100, Millwood, NY 10546.

The Pacific Journal of Mathematics ISSN 0030-8730 is published monthly by the Pacific Journal of Mathematics at P.O. Box 969, Carmel Valley, CA 93924. Application to mail at Second-class postage rates is pending at Carmel Valley, California, and additional mailing offices. Postmaster: Send address changes to Pacific Journal of Mathematics, P. O. Box 969, Carmel Valley, CA 93924.

PUBLISHED BY PACIFIC JOURNAL OF MATHEMATICS, A NON-PROFIT CORPORATION

Copyright $\odot 1983$ by Pacific Journal of Mathematics 


\section{Pacific Journal of Mathematics}

Vol. 104, No. $2 \quad$ June, 1983

Leo James Alex, Simple groups and a Diophantine equation ........... 257

Herbert James Alexander and John Wermer, On the approximation of

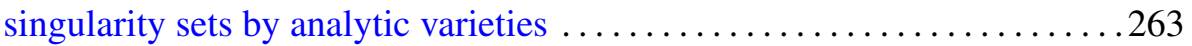

Waleed A. Al-Salam and Mourad Ismail, Orthogonal polynomials associated with the Rogers-Ramanujan continued fraction .......... 269

J. L. Brenner and Roger Conant Lyndon, Permutations and cubic

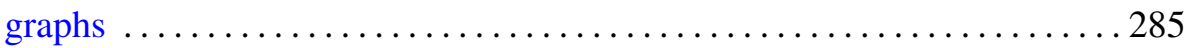

Ian George Craw and Susan Ross, Separable algebras over a commutative

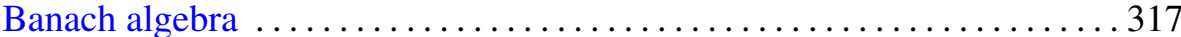

Jesus M. Dominguez, Non-Archimedean Gel'fand theory ............. 337

David Downing and Barry Turett, Some properties of the characteristic of

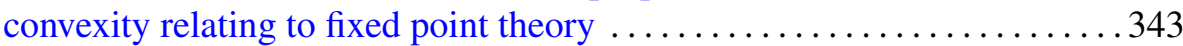

James Arthur Gerhard and Mario Petrich, Word problems for free objects in certain varieties of completely regular semigroups $\ldots \ldots \ldots 351$

Moses Glasner and Mitsuru Nakai, Surjective extension of the reduction

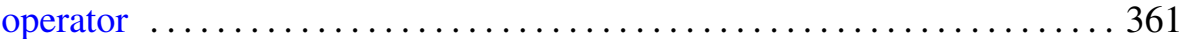

Takesi Isiwata, Ultrafilters and mappings $\ldots \ldots \ldots \ldots \ldots \ldots \ldots \ldots \ldots \ldots \ldots$

Lowell Duane Loveland, Double tangent ball embeddings of curves in $E^{3}$

Douglas C. McMahon and Ta-Sun Wu, Homomorphisms of minimal flows

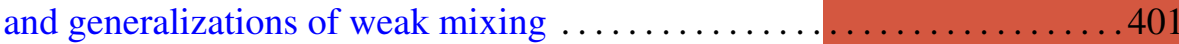

P. H. Maserick, Applications of differentiation of $\mathscr{L}_{p}$-functions to semilattices

Wayne Bruce Powell and Constantine Tsinakis, Free products in the class of abelian $l$-groups

Bruce Reznick, Some inequalities for products of power sums

C. Ray Rosentrater, Compact operators and derivations induced by

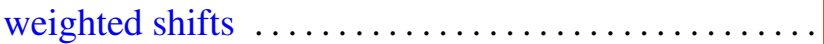

Edward Silverman, Basic calculus of variations

Charles Andrew Swanson, Criteria for oscillatory sublinear Schrödinger equations

David J. Winter, The Jacobson descent theorem 